\title{
Comprehensive Comparisons Between Six-Arm and Nine-Arm Modular Multilevel Converter
}

\author{
Futian Qin, Feng Gao, Yi Tang, Jinyu Wang, and Tao Xu
}

\begin{abstract}
Nine-arm modular multilevel converter (9A-MMC) is a compact modular multilevel converter (MMC) with two sets of three-phase output terminals, and it can be regarded as the integration of two conventional six-arm modular multilevel converters (6A-MMC) with double function middle arms. Compared with two 6A-MMCs, the required submodules (SMs) in 9A-MMC can be reduced, however, there will be some performance tradeoffs, and the performance tradeoffs of 9A-MMC have not yet been investigated in detail. Hence, this paper gives the comprehensive comparisons between 6A-MMC and 9A-MMC, including the required SMs number, the current stress of the semiconductor device, the maximum capacitor voltage ripple, and the power losses of the whole system. This paper can be a guide to make the best use of the advantages and avoid the disadvantages of 9A-MMC. Finally, the simulation results verify the mathematical analysis of the comparisons.
\end{abstract}

Index Terms - Capacitor voltage ripple, current stress, nine-arm MMC, power losses, six-arm MMC, submodules number.

\section{INTRODUCTION}

$\mathrm{S}^{\mathrm{n}}$ INCE modular multilevel converter (MMC) was first proposed in [1], [2], it has drawn the increasing interests and become one of the most promising multilevel topologies in medium/high-voltage applications, such as high-voltage direct current (HVDC) transmission systems [3]-[5], flexible AC transmission systems (FACTs) [6]-[8], medium-voltage motor drives [9], [10], etc., due to its prominent advantages of modularity, scalability, and superior harmonic performances [11]-[13].

There are several medium/high-voltage applications including

Manuscript received January 15, 2020; revised June 4, 2020; accepted June 11, 2020. Date of publication June 30, 2020; date of current version June 19, 2020. This work was supported in part by the National Natural Science Foundation of China under Grant 51722704, in part by the Shandong Provincial Natural Science Foundation, China, under Grant JQ201717, in part by the Foundation for Innovative Research Groups of National Natural Science Foundation of China, under Grant 61821004, and in part by Shandong Provincial Key Research and Development Program (Major Scientific and Technological Innovation Project), under Grant 2019JZZY020805. (Corresponding author: Feng Gao.)

F. Qin, F. Gao, and T. Xu are with Key Laboratory of Power System Intelligent Dispatch and Control of Ministry of Education, Jinan 250061, China (e-mail: ft_qin@163.com; fgao@sdu.edu.cn; xutaojason@163.com).

J. Wang and Y. Tang are with the School of Electrical and Electronic Engineering, Nanyang Technological University, Singapore 639798 (e-mail: wangjy@ntu.edu.sg; yitang@ntu.edu.sg).

Digital Object Identifier 10.24295/CPSSTPEA.2020.00012 two sets of three-phase terminals, such as medium-voltage dual-motor drives, unified power flow controller (UPFC), where two independent MMCs were generally employed [8], [14]. Fortunately, it is possible to integrate two MMCs into one compact nine-arm MMC (9A-MMC) with two sets of threephase terminals [15]-[18]. Compared with two 6A-MMCs, $9 \mathrm{~A}-\mathrm{MMC}$ can save $25 \%$ of the required arms and $50 \%$ of the arm inductors, and the corresponding SM capacitors, SM capacitor voltage sensors, gate-drive circuits, and arm current sensors can also be reduced. This can significantly reduce system weight, volume, and cost. Meanwhile, the 6A-MMC's excellent advantages of modularity, scalability, high reliability and superior harmonic performance can be inherited in 9A-MMC.

The 9A-MMC was firstly proposed in [19], where the submodules (SMs) was powered by the ideal DC-source to simplify the control scheme design, and the circulating current suppression control (CCSC) and capacitor voltage balancing control (CVBC) were not considered. The detailed operation analysis and control scheme of 9A-MMC were firstly presented in [20]-[21]. 9A-MMC is topologically similar to the nine-switch converter, which was first proposed in [22]. Although the nine-switch converter can save components in configuration, the performance tradeoffs are always unavoidable. For instance, there are strict restrictions in its total attainable amplitude and phase shift between its two terminal sets, and the dc-link voltage would be higher compared with that of conventional back-to-back voltage source converter (VSC) in some operating scenarios [23]. Many previous studies have investigated the performance tradeoffs of the nine-switch converter and given the comparisons between the nine-switch converter and twelve-switch converter, including the current stress [24] and power losses [25], [26]. It has to be pointed out that the existing researches only emphasize the 9A-MMC's advantage of reducing SMs number, however, the performance tradeoffs of 9A-MMC have not yet been reported and investigated in detail. One of the performance tradeoffs of 9A-MMC is that the operation range of 9A-MMC is limited, and the modulation ratios of two sets of three-phase terminals and the relative phase angle between them cannot be set arbitrarily. Fortunately, a unique operational mode of 9A-MMC that the number of SMs in each arm can be different was presented in [21], and the operation range limitation of 9A-MMC can be eliminated by the elaborate design of the number of SMs in each arm. Under this unique operation 


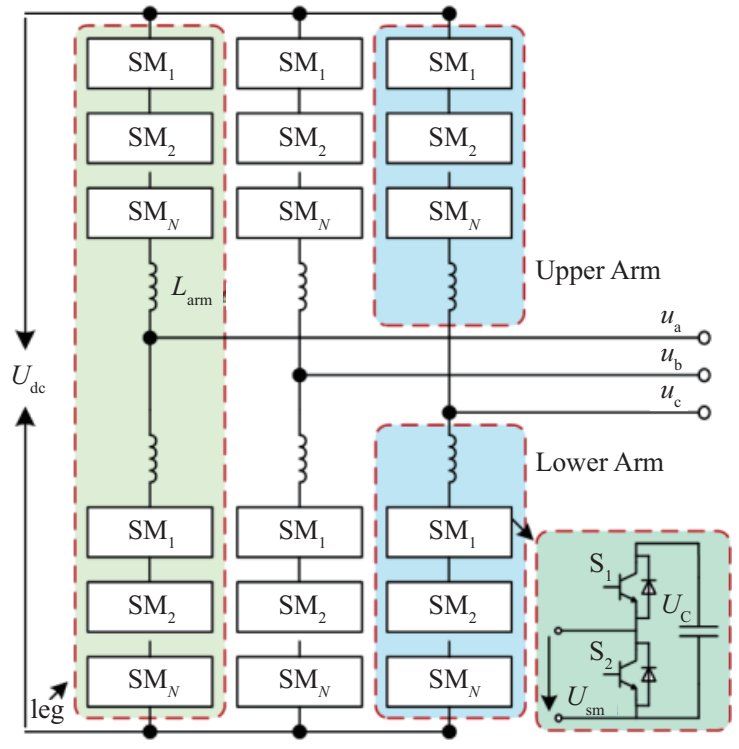

Fig. 1. Topology of the conventional six-arm modular multilevel converter (6A-MMC).

mode, the DC-side voltage of 9A-MMC needs to be varied with the sum of SM number in one phase. Moreover, there are the other performance tradeoffs of 9A-MMC should be fully analyzed.

Therefore, this paper gives comprehensive comparisons between two conventional 6A-MMCs and 9A-MMC, including the required SM number, the current stress of the semiconductor device, the maximum capacitor voltage ripple, and the power losses of the whole system. The simulation results finally verify the corresponding mathematical analysis.

\section{Topologies and Mathematical Models of 6A-MMC AND 9A-MMC}

\section{A. Topologies of $6 A-M M C$ and $9 A-M M C$}

Fig. 1 illustrates the conventional 6A-MMC, which is composed of three phase-legs, where each phase-leg consists of two arms and each arm comprises $N$ series-connected halfbridge SMs and a series arm inductor $L_{\text {arm }}$. The 9A-MMC is depicted in Fig. 2, and the 9A-MMC also consists of three phase-legs, but each phase-leg is formed by three arms, named as the upper, middle, and lower arm, respectively. The upper, middle, and lower arm separately comprises $k_{1}, k_{2}$, and $k_{3}$ halfbridge SMs, $L_{\mathrm{u}}$ and $L_{1}$ are the arm inductors in upper and lower arm with $L_{\mathrm{u}}=L_{\mathrm{l}}$. Being different, there is only one set of threephase terminal in conventional 6A-MMC, which is located at the middle point between upper and lower arm, while there are two sets of three-phase terminals in 9A-MMC, one is located at the middle point between upper and middle arm, and the other is located at the middle point between middle and lower arm. Moreover, the SMs number of upper and lower arm in 6A-MMC are usually equal, but the SMs number of upper, middle, and lower arm in 9A-MMC can be unequal.

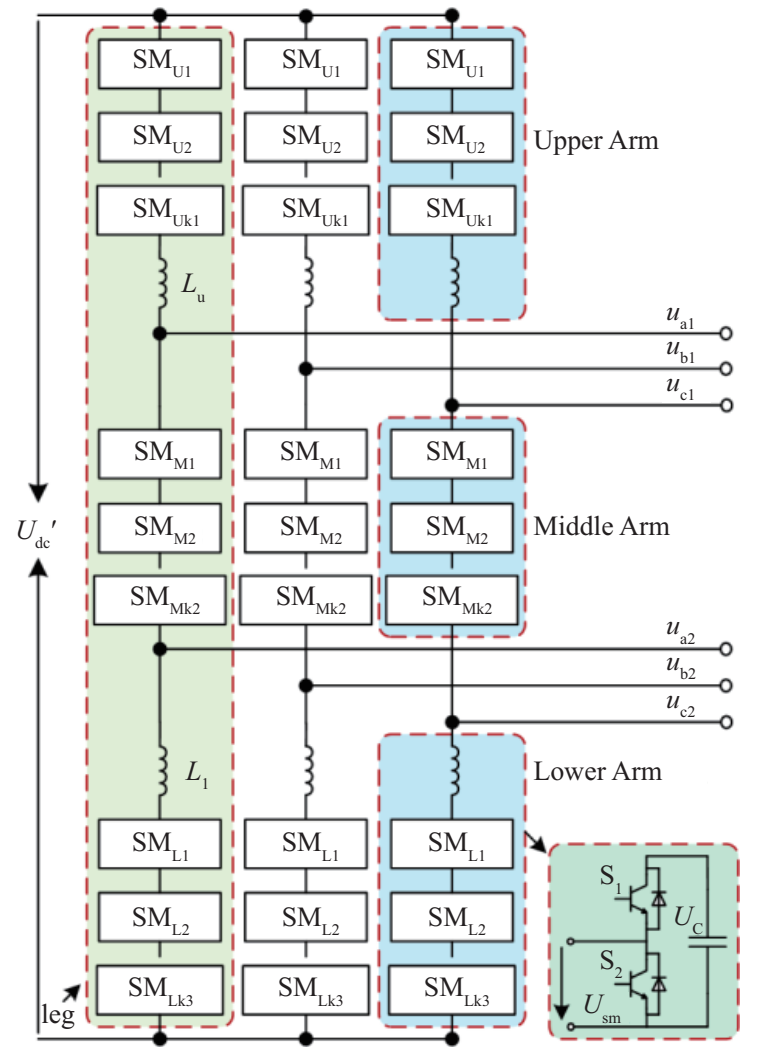

Fig. 2. Topology of the nine-arm modular multilevel converter (9A-MMC).

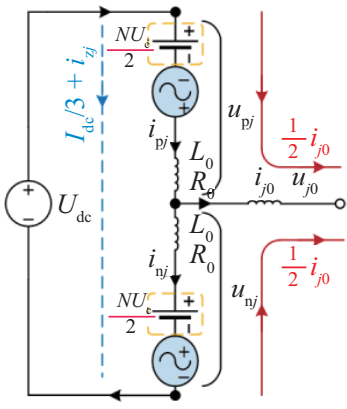

(a)

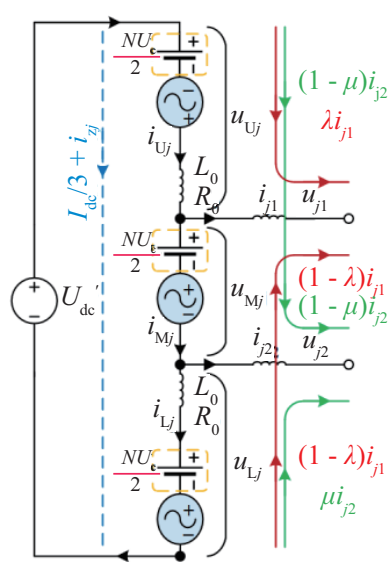

(b)
Fig. 3. Single-phase equivalent models of (a) the 6A-MMC, (b) the 9A-MMC.

\section{B. Mathematical Models of 6A-MMC and 9A-MMC}

The simplified equivalent models of single-phase conventional 6A-MMC and 9A-MMC are depicted in Fig. 3 (a) and (b), where the SMs are considered as the controlled voltage sources and the voltage of each arm is the combination of these controlled voltage sources. Because the upper and lower arm in 6A-MMC are generally symmetrical, the output current will be divided equally between upper and lower arm. Therefore, according to the equivalent model, the mathematical expressions of arms' voltages and currents in $j(j=\mathrm{a}, \mathrm{b}$, or $\mathrm{c})$ 
phase of 6A-MMC can be expressed as follows:

$$
\begin{gathered}
u_{\mathrm{pj}}=\frac{1}{2} N U_{\mathrm{c}}-u_{j 0}-\left(L_{0} \frac{\mathrm{d} i_{\mathrm{pj}}}{\mathrm{d} t}+R_{0} i_{\mathrm{pj}}\right) \\
u_{\mathrm{nj}}=\frac{1}{2} N U_{\mathrm{c}}+u_{j 0}-\left(L_{0} \frac{\mathrm{d} i_{\mathrm{nj}}}{\mathrm{d} t}+R_{0} i_{\mathrm{nj}}\right) \\
i_{\mathrm{p} j}=\frac{1}{3} I_{\mathrm{dc}}+\frac{1}{2} i_{j 0}+\sum_{n=2}^{+\infty} i_{z j}^{(n)} \\
i_{\mathrm{n} j}=\frac{1}{3} I_{\mathrm{dc}}-\frac{1}{2} i_{j 0}+\sum_{n=2}^{+\infty} i_{z j}^{(n)}
\end{gathered}
$$

where $u_{\mathrm{p} j}$ and $u_{\mathrm{n} j}$ are the upper and lower arm voltage in conventional $6 \mathrm{~A}-\mathrm{MMC}, i_{\mathrm{p} j}$ and $i_{\mathrm{n} j}$ are the upper and lower arm current, $N$ is the number of SMs per arm, $U_{\mathrm{c}}$ is the rated $\mathrm{SM}$ capacitor voltage, $u_{j 0}$ and $i_{j 0}$ are the output voltage and current, $I_{\mathrm{dc}}$ is the DC-side current, $i_{z j}{ }^{(n)}$ is the $n$ th-order circulating current, $L_{0}$ and $R_{0}$ are the inductance and resistance of arm inductor.

In 9A-MMC, each phase-leg is formed by three arms, and the upper and lower output currents can be coupled via the middle arm. Besides, because of the asymmetry of three arms in the same phase-leg, the division of the upper and lower output currents among three arms will be unequal. The mathematical expressions of arms' voltages and currents in $j(j=\mathrm{a}, \mathrm{b}$, or $\mathrm{c})$ phase of 9A-MMC can be expressed as follows:

$$
\begin{gathered}
u_{\mathrm{U} j}=\frac{1}{2} k_{1} U_{\mathrm{c}}-u_{j 1}-\left(L_{0} \frac{\mathrm{d} i_{\mathrm{U} j}}{\mathrm{~d} t}+R_{0} i_{\mathrm{U} j}\right) \\
u_{\mathrm{M} j}=\frac{1}{2} k_{2} U_{\mathrm{c}}+u_{j 1}-u_{j 2} \\
u_{\mathrm{L} j}=\frac{1}{2} k_{3} U_{\mathrm{c}}+u_{j 2}-\left(L_{0} \frac{\mathrm{d} i_{\mathrm{I} j}}{\mathrm{~d} t}+R_{0} i_{\mathrm{L} j}\right) \\
i_{\mathrm{U} j}=\frac{1}{3} I_{\mathrm{dc}}+\lambda i_{j 1}+(1-\mu) i_{j 2}+\sum_{n=2}^{+\infty} i_{z j}^{(n)} \\
i_{\mathrm{M} j}=\frac{1}{3} I_{\mathrm{dc}}-(1-\lambda) i_{j 1}+(1-\mu) i_{j 2}+\sum_{n=2}^{+\infty} i_{z j}^{(n)} \\
i_{\mathrm{I} j}=\frac{1}{3} I_{\mathrm{dc}}-(1-\lambda) i_{j 1}-\mu i_{j 2}+\sum_{n=2}^{+\infty} i_{z j}^{(n)}
\end{gathered}
$$

where $u_{\mathrm{U} j}, u_{\mathrm{M} j}$, and $u_{\mathrm{L} j}$ are the upper, middle, and lower arm voltages in 9A-MMC, and $i_{\mathrm{U} j}, i_{\mathrm{M} j}$, and $i_{\mathrm{Lj}}$ are the upper, middle, and lower arm currents, $u_{j 1}, u_{j 2}, i_{j 1}$, and $i_{j 2}$ are the upper and lower output voltages and currents, $k_{1}, k_{2}$, and $k_{3}$ are the number of SMs in upper, middle, and lower arm, $\lambda$ and $\mu$ are the distribution coefficients of upper and lower output currents among three arms.

The DC-side voltage of the conventional 6A-MMC and 9A-MMC can be derived as follows:

$$
\begin{gathered}
U_{\mathrm{dc} \_6 \mathrm{~A}-\mathrm{MMC}}=N U_{\mathrm{c}} \\
U_{\mathrm{dc} \_9 \mathrm{~A}-\mathrm{MMC}}=\frac{\left(k_{1}+k_{2}+k_{3}\right) U_{\mathrm{c}}}{2} .
\end{gathered}
$$

\section{Comparisons Between 6A-MMC and 9A-MMC}

The original intention of $9 \mathrm{~A}-\mathrm{MMC}$ is to realize the same function as two 6A-MMCs, therefore, the following comparisons should be carried out between two 6A-MMCs and 9A-MMC. To distinguish two 6A-MMCs, they are named as 6A-MMC $\mathrm{An}_{1}$ and $6 \mathrm{~A}-\mathrm{MMC}_{2}$, respectively.

\section{A. Required SMs Number}

The output phase voltages of two 6A-MMCs can be assumed as follows:

$$
\begin{gathered}
u_{j 1}(t)=U_{j 1} \sin (\omega t) \\
u_{j 2}(t)=U_{j 2} \sin (\omega t+\theta)
\end{gathered}
$$

where $U_{j 1}$ and $U_{j 2}$ are the amplitude of output phase voltages in $6 \mathrm{~A}-\mathrm{MMC}_{1}$ and $6 \mathrm{~A}-\mathrm{MMC}_{2}$, respectively, $\theta$ is the relative phase angle of output phase voltages between $6 \mathrm{~A}-\mathrm{MMC}_{1}$ and $6 \mathrm{~A}-\mathrm{MMC}_{2}$. The comparisons should be under the same operation condition, hence $U_{j 1}$ and $U_{j 2}$ can also represent the amplitude of upper and lower output phase voltages in 9A-MMC, $\theta$ is the relative phase angle between upper and lower output phase voltages in 9A-MMC.

The modulation ratios of output terminals in $6 \mathrm{~A}-\mathrm{MMC}_{1}$ and $6 \mathrm{~A}-\mathrm{MMC}_{2}$ can be separately defined as follows:

$$
\begin{aligned}
& M_{1}=\frac{2 U_{j 1}}{N U_{c}} \\
& M_{2}=\frac{2 U_{j 2}}{N U_{c}} .
\end{aligned}
$$

The number $N$ of SMs per arm in 6A-MMC can be calculated as follow:

$$
N=\frac{U_{\mathrm{dc} \_6 \mathrm{~A}-\mathrm{MMC}}}{U_{\mathrm{c}}} .
$$

The arm reference voltages of 9A-MMC can be calculated by (5)-(7) and (13)-(14), and neglecting the voltages of arm inductors, the normalized arm reference voltages of 9A-MMC can be expressed as follows:

$$
\begin{gathered}
u_{\mathrm{U} j}(t)=\frac{1}{2}\left[1-M_{1}^{\prime} \sin (\omega t)\right] \\
u_{\mathrm{M} j}(t)=\frac{1}{2}\left[1+\frac{k_{1}}{k_{2}} M_{1}^{\prime} \sin (\omega t)-\frac{k_{3}}{k_{2}} M_{2}^{\prime} \sin (\omega t+\theta)\right](19) \\
u_{\mathrm{L} j}(t)=\frac{1}{2}\left[1+M_{2}^{\prime} \sin (\omega t+\theta)\right]
\end{gathered}
$$

where $M_{1}^{\prime}=2 U_{j 1} / k_{1} U_{\mathrm{c}}$ and $M_{2}^{\prime}=2 U_{j 2} / k_{3} U_{\mathrm{c}}$ are the modulation ratios of upper and lower output terminals in 9A-MMC.

According to the unique operation model of 9A-MMC 


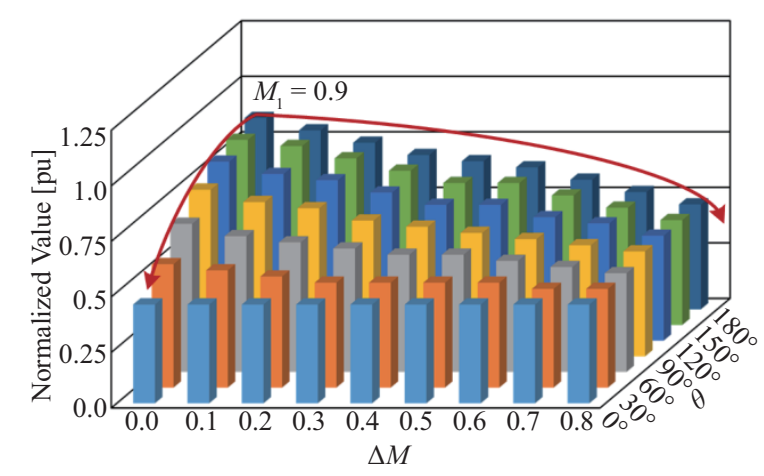

Fig. 4. The number of required SMs in 9A-MMC with the change of amplitude difference and relative phase angle.

presented in [21], the SMs number in each arm can be different. The number of SMs in upper arm $k_{1}$ depends on the amplitude of the upper output phase voltage and the rated SM capacitor voltage, while the number of SMs in lower arm $k_{3}$ depends on the amplitude of the lower output phase voltage and the rated SM capacitor voltage. Their expressions can be given as follows:

$$
\begin{aligned}
& k_{1}=\text { ceil }\left[\frac{2 U_{j 1}}{U_{c}}\right] \\
& k_{3}=\operatorname{ceil}\left[\frac{2 U_{j 2}}{U_{\mathrm{c}}}\right]
\end{aligned}
$$

where ceil is the top integral function. Being different, the number of SMs in the middle arm $k_{2}$ depends on not only the amplitude of both upper and lower output phase voltages but also the relative phase angle between two terminals. $k_{2}$ can be expressed as follow:

$$
k_{2}=\operatorname{ceil}\left[\sqrt{\left(k_{1} M_{1}^{\prime}-k_{3} M_{2}^{\prime} \cos \theta\right)^{2}+\left(k_{3} M_{2}^{\prime} \sin \theta\right)^{2}}\right] .
$$

To demonstrate the advantage of 9A-MMC for reducing the SMs number, Fig. 4 shows the graphic comparisons of the required SMs number between two 6A-MMCs and 9A-MMC under different modulation ratio difference $\triangle M$ and relative phase angle $\theta$, where $\Delta M=M_{1}-M_{2}$, and $M_{1}$ is set as 0.9 . It is noted that the number of SMs in two 6A-MMCs is taken as the reference for comparison. Besides the SMs number comparisons, these comparisons can also represent the number of the SM capacitors, IGBTs, diodes, gate-drive circuits, or SM capacitor voltage sensors comparisons, because these comparisons have been normalized. As seen, compared with two 6A-MMCs, the number of required components in 9A-MMC can be dramatically reduced when the modulation ratio difference $\Delta M$ is large and the relative phase angle is small, but with the modulation ratio difference $\Delta M$ becoming small and the relative phase angle increasing, the advantage

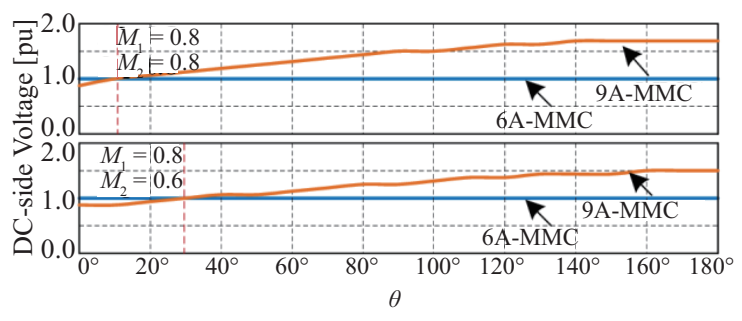

Fig. 5. Comparison of the DC-side voltage between the 6A-MMC and 9A-MMC under the different operation conditions.

of 9A-MMC for reducing components will not be obvious. The comparisons of the DC-side voltage in the 6A-MMC and 9A-MMC under the different operation conditions are shown in Fig. 5 where the DC-side voltage in the $6 \mathrm{~A}-\mathrm{MMC}$ is taken as the reference. As shown in Fig. 5, the DC-side voltage in the $9 \mathrm{~A}-\mathrm{MMC}$ is not always higher than that in the 6A-MMC, and the DC-side voltage in the 9A-MMC may be lower than that in the $6 \mathrm{~A}-\mathrm{MMC}$ when $\theta$ is small.

The above comparisons are based on the operation model that the operation frequencies of two output terminals are identical. If the operation frequencies of two output terminals are different, the expression of the number of SMs in the middle arm shown in (23) will not be suitable. Because the middle arm voltage should be larger than zero and less than $k_{2} U_{\mathrm{c}}$, hence, the number of SM in middle arm $k_{2}$ should satisfy (24) under different frequency operation mode.

$$
\begin{aligned}
& k_{2}=\operatorname{Max}\{ \operatorname{Max}\left[k_{1} M_{1}^{\prime} \sin \left(\omega_{1} t\right)-k_{3} M_{2}^{\prime} \sin \left(\omega_{2} t+\theta\right)\right], \\
&\left.\operatorname{Max}\left[k_{3} M_{2}^{\prime} \sin \left(\omega_{2} t+\theta\right)-k_{1} M_{1}^{\prime} \sin \left(\omega_{1} t\right)\right]\right\}
\end{aligned}
$$

Under the specific different frequency operation mode, the number of the required SM in 9A-MMC when $\left(M_{1}, M_{2}\right)$ are set as $(0.8,0.8), \theta$ is set as $60^{\circ}$ or $90^{\circ}$ are normalized and listed in Table I and II, respectively, where the number of required SMs under the common frequency operation mode is taken as the reference. Compared with the common frequency operation mode, the different frequency operation mode will need more SMs, which can weaken the advantage of 9A-MMC for reducing components. Therefore, the different frequency operation mode will not be elaborated in the following sections.

\section{B. Current Stress of Semiconductor Device}

The current stress of the semiconductor device is one of the standards for semiconductor device selection, which is significantly important to the operation safety of the converter. To determine the current stress of the semiconductor device, the arm currents should be firstly calculated, and the maximum of arm currents can be regarded as the current stress of the semiconductor device. The arm currents in 6A-MMC can be easily calculated by (3) and (4), however, to obtain the arm currents in 9A-MMC, the distribution coefficients $(\lambda, \mu)$ of upper and lower output currents among three arms should be 
TABLE I

The Number of Reguired SM in 9A-MMC Under Different Frequency Operation Model $\left(M_{1}=M_{2}=0.8, \theta=60^{\circ}\right)$

\begin{tabular}{ccccccccc}
\hline \hline$\frac{\omega_{1(\mathrm{~Hz})}}{\omega_{2(\mathrm{~Hz})}}$ & 25 & 50 & 75 & 100 & 125 & 150 & 175 & 200 \\
\hline 25 & 1 & $9 / 7$ & $8 / 7$ & $9 / 7$ & $9 / 7$ & $9 / 7$ & $9 / 7$ & $9 / 7$ \\
50 & $9 / 7$ & 1 & $9 / 7$ & $9 / 7$ & $9 / 7$ & $8 / 7$ & $9 / 7$ & $9 / 7$ \\
75 & $9 / 7$ & $9 / 7$ & 1 & $9 / 7$ & $9 / 7$ & $9 / 7$ & $9 / 7$ & $9 / 7$ \\
100 & $9 / 7$ & $9 / 7$ & $9 / 7$ & 1 & $9 / 7$ & $9 / 7$ & $9 / 7$ & $9 / 7$ \\
125 & $9 / 7$ & $9 / 7$ & $26 / 21$ & $9 / 7$ & 1 & $9 / 7$ & $9 / 7$ & $9 / 7$ \\
150 & $9 / 7$ & $9 / 7$ & $9 / 7$ & $9 / 7$ & $9 / 7$ & 1 & $9 / 7$ & $9 / 7$ \\
175 & $9 / 7$ & $9 / 7$ & $9 / 7$ & $9 / 7$ & $9 / 7$ & $26 / 21$ & 1 & $9 / 7$ \\
200 & $9 / 7$ & $9 / 7$ & $9 / 7$ & $9 / 7$ & $9 / 7$ & $9 / 7$ & $9 / 7$ & 1 \\
\hline \hline
\end{tabular}

TABLE II

The Number of Required SM in 9A-MMC Under DifFerent Frequency Operation Model $\left(M_{1}=M_{2}=0.8, \theta=90^{\circ}\right)$

\begin{tabular}{ccccccccc}
\hline \hline$\frac{\omega_{1(\mathrm{~Hz})}}{\omega_{2(\mathrm{~Hz})}}$ & 25 & 50 & 75 & 100 & 125 & 150 & 175 & 200 \\
\hline 25 & 1 & $13 / 12$ & $9 / 8$ & $9 / 8$ & $9 / 8$ & $9 / 8$ & $9 / 8$ & $9 / 8$ \\
50 & $9 / 8$ & 1 & $9 / 8$ & $13 / 12$ & $9 / 8$ & $9 / 8$ & $9 / 8$ & $9 / 8$ \\
75 & $9 / 8$ & $9 / 8$ & 1 & $9 / 8$ & $9 / 8$ & $13 / 12$ & $9 / 8$ & $9 / 8$ \\
100 & $9 / 8$ & $9 / 8$ & $9 / 8$ & 1 & $9 / 8$ & $9 / 8$ & $9 / 8$ & $13 / 12$ \\
125 & $9 / 8$ & $9 / 8$ & $9 / 8$ & $9 / 8$ & 1 & $9 / 8$ & $9 / 8$ & $9 / 8$ \\
150 & $9 / 8$ & $9 / 8$ & $9 / 8$ & $9 / 8$ & $9 / 8$ & 1 & $9 / 8$ & $9 / 8$ \\
175 & $9 / 8$ & $9 / 8$ & $9 / 8$ & $9 / 8$ & $9 / 8$ & $9 / 8$ & 1 & $9 / 8$ \\
200 & $9 / 8$ & $9 / 8$ & $9 / 8$ & $9 / 8$ & $9 / 8$ & $9 / 8$ & $9 / 8$ & 1 \\
\hline \hline
\end{tabular}

firstly calculated.

The output currents of 9A-MMC can be assumed as follows:

$$
\begin{gathered}
i_{j 1}(t)=I_{j 1} \sin \left(\omega t-\varphi_{1}\right) \\
i_{j 2}(t)=I_{j 2} \sin \left(\omega t+\theta-\varphi_{2}\right)
\end{gathered}
$$

where $I_{j 1}$ and $I_{j 2}$ are the amplitude of upper and lower output currents, $\varphi_{1}$ and $\varphi_{2}$ are the power factor angle of upper and lower output terminal in 9A-MMC. Meanwhile, $I_{j 1}$ and $I_{j 2}$ can also refer to the amplitude of output currents in $6 \mathrm{~A}-\mathrm{MMC}_{1}$ and $6 \mathrm{~A}-\mathrm{MMC}_{2}$, respectively, $\varphi_{1}$ and $\varphi_{2}$ can be the power factor angle of output terminals in $6 \mathrm{~A}-\mathrm{MMC}_{1}$ and $6 \mathrm{~A}-\mathrm{MMC}_{2}$.

According to (5) $-(7)$ and (13)-(14), the arm reference voltages in 9A-MMC can be expressed as follows:

$$
\begin{gathered}
u_{\mathrm{U} j \_ \text {ref }}=\frac{1}{2} k_{1} U_{\mathrm{c}}-U_{j 1} \sin (\omega t) \\
u_{\mathrm{M} j \_ \text {ref }}=\frac{1}{2} k_{2} U_{\mathrm{c}}+U_{j 1} \sin (\omega t)-U_{j 2} \sin (\omega t+\theta) \\
u_{\mathrm{L} j_{j} \text { ref }}=\frac{1}{2} k_{3} U_{\mathrm{c}}+U_{j 2} \sin (\omega t+\theta) .
\end{gathered}
$$

According to (8)-(10) and (25)-(26), and assuming the circulating current to be suppressed effectively, the arm currents of 9A-MMC can be expressed as follows:

$$
i_{\mathrm{Uj}}=\frac{1}{3} I_{\mathrm{dc}}+\lambda I_{j 1} \sin \left(\omega t-\varphi_{1}\right)+(1-\mu) I_{j 2} \sin \left(\omega t+\theta-\varphi_{2}\right)
$$

$$
\begin{gathered}
i_{\mathrm{Mj}}=\frac{1}{3} I_{\mathrm{dc}}-(1-\lambda) I_{j 1} \sin \left(\omega t-\varphi_{1}\right)+(1-\mu) I_{j 2} \sin \left(\omega t+\theta-\varphi_{2}\right) \\
i_{\mathrm{L} j}=\frac{1}{3} I_{\mathrm{dc}}-(1-\lambda) I_{j 1} \sin \left(\omega t-\varphi_{1}\right)-\mu I_{j 2} \sin \left(\omega t+\theta-\varphi_{2}\right)
\end{gathered}
$$

The average absorbed power of each arm can be calculated by multiplying corresponding arm voltage with arm current, which can be expressed as follows:

$$
\begin{aligned}
P_{\mathrm{U} j} & =\frac{1}{T} \int_{0}^{T} u_{\mathrm{L} j} \times i_{\mathrm{Lj}} \mathrm{d} t \\
& =\frac{1}{T} \int_{0}^{T}\left(\frac{1}{2} k_{1} U_{\mathrm{c}}-u_{j 1}\right) \times\left[\frac{1}{3} I_{\mathrm{dc}}+\lambda i_{j 1}+(1-\mu) i_{j 2}\right] \mathrm{d} t \\
P_{\mathrm{v} j} & =\frac{1}{T} \int_{0}^{T} u_{\mathrm{w} j} \times i_{\mathrm{uj}} \mathrm{d} t \\
& =\frac{1}{T} \int_{0}^{T}\left(\frac{1}{2} k_{2} U_{\mathrm{c}}+u_{j 1}-u_{j 2}\right) \times\left[\frac{1}{3} I_{\mathrm{dc}}-(1-\lambda) i_{j 1}+(1-\mu) i_{j 2}\right] \mathrm{d} t \\
P_{\mathrm{L} j} & =\frac{1}{T} \int_{0}^{T} u_{\mathrm{L} j} \times i_{\mathrm{L} j} d t \\
& =\frac{1}{T} \int_{0}^{T}\left(\frac{1}{2} k_{3} U_{\mathrm{c}}+u_{j 2}\right) \times\left[\frac{1}{3} I_{\mathrm{dc}}-(1-\lambda) i_{j 1}-\mu i_{j 2}\right] \mathrm{d} t .
\end{aligned}
$$

The detailed expression of $p_{\mathrm{U} j}, p_{\mathrm{M} j}$, and $p_{\mathrm{L} j}$ are shown in (A1), (A2), and (A3), respectively. Moreover, the expression of the DC-side current is shown in (A4). Under steady operation state, the transferred active power between DC-side and $\mathrm{AC}$-side is equal, which means that the upper, middle, and lower arm absorb no active power and the absorbed active power by three arms should satisfy (36)-(38).

$$
\begin{aligned}
P_{\mathrm{L} j} & =0 \\
P_{\mathrm{M} j} & =0 \\
P_{\mathrm{L} j} & =0
\end{aligned}
$$

(36)-(38) can compose a set of equations about $\lambda$ and $\mu$, and the values of $\lambda$ and $\mu$ can be determined by solving these equations.

Fig. 6(a) and (b) display the comparisons of the current stress of semiconductor device between two 6A-MMCs and 9A-MMC when the modulation ratios $\left(M_{1}, M_{2}\right)$ are set as 0.8 and $0.8,0.8$ and 0.6 , respectively, and the relative phase angle $\theta$ changes from $0^{\circ}$ to $180^{\circ}$. It is shown that the current stress of the semiconductor device in 9A-MMC may be larger than that of 6A-MMC, but this case is not universal. The current stress of the semiconductor device in $9 \mathrm{~A}-\mathrm{MMC}$ can be almost equivalent to that in $6 \mathrm{~A}-\mathrm{MMC}$ when the modulation ratios are set as 0.8 and 0.8 , and the relative phase angle is from $110^{\circ}$ to 


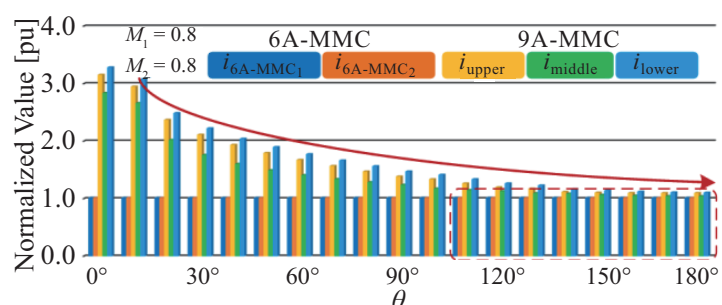

(a)

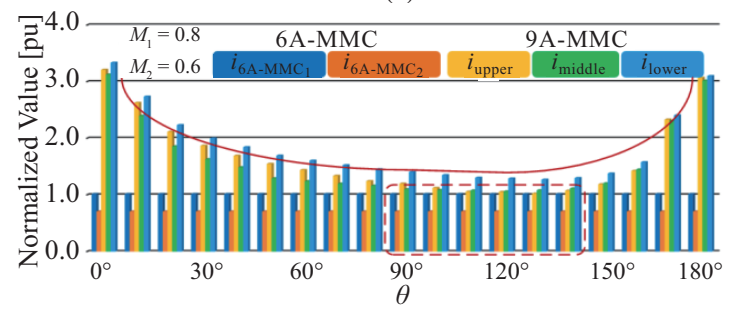

(b)

Fig. 6. Comparisons of the current stress of the semiconductor device between two 6A-MMCs and 9A-MMC when the modulation ratios are set as (a) 0.8 and 0.8 , (b) 0.8 and 0.6 .

$180^{\circ}$. However, in the case that the modulation ratios are set as 0.8 and 0.6 , the range of the relative phase angle is from $90^{\circ}$ to $140^{\circ}$ when the current stress of the semiconductor device in 9A-MMC can be almost equivalent to that in 6A-MMC. Therefore, the modulation ratios of two sets of output terminals and the relative phase angle between them have a significant influence on the current stress of semiconductor in 9A-MMC. To avoid the large current stress of the semiconductor device in 9A-MMC, the operation scenario of 9A-MMC should be selected elaborately. To investigate the influence of the power factor angle $\varphi$ on the current stress of the semiconductor devices in 6A-MMC and 9A-MMC, the comparisons of the current stress of the semiconductor devices between the 6A-MMC and 9A-MMC with different $\varphi$ are shown in Fig. 7(a) and (b), respectively. As shown in Fig. 7, the current stress of the semiconductor device in the 6A-MMC is minimum when $\varphi$ is $90^{\circ}$ and $270^{\circ}$ (i.e., the output active power is equal to 0 ). The current stress of the semiconductor device of the middle arm in the $9 \mathrm{~A}-\mathrm{MMC}$ is also minimum when $\varphi$ is $90^{\circ}$ and $270^{\circ}$, but $\varphi$ is not $90^{\circ}$ or $270^{\circ}$ when the current stress of semiconductor devices of the upper and lower arms in 9A-MMC is minimum, which varies with the operation conditions.

\section{Maximum SM Capacitor Voltage Ripple}

In [27], the SM capacitor voltage ripples in conventional 6A-MMC have been investigated in detail, and the SM capacitor voltage ripples in 6A-MMC mainly consist of the first- and second-order ripple components. The maximum SM capacitor voltage ripple in 6A-MMC can be obtained according to [27]. Neglecting the high-frequency components, the switching functions of upper, middle, and lower arm in 9A-MMC can be defined as follows:

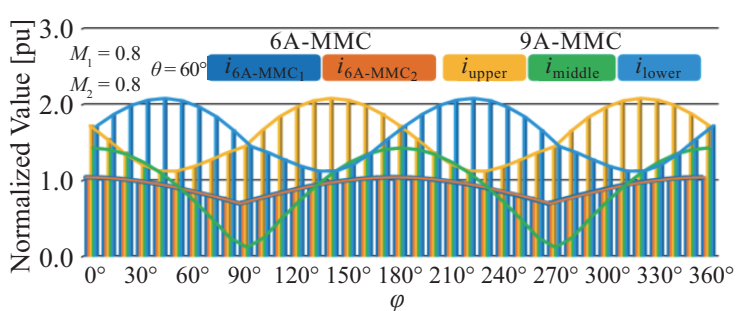

(a)

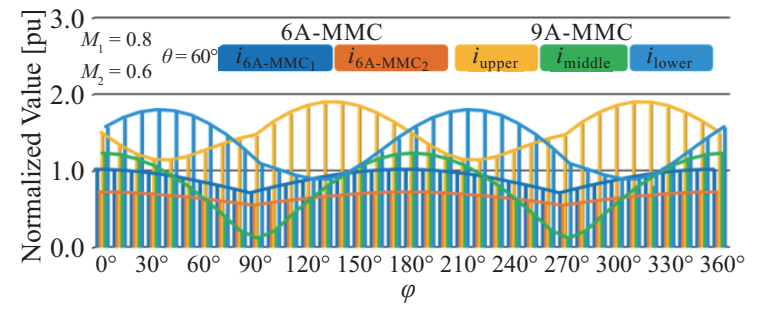

(b)

Fig. 7. Comparisons of the current stress of the semiconductor device between two 6A-MMCs and 9A-MMC when $\left(M_{1}, M_{2}\right)$ and $\theta$ are set as (a) $(0.8,0.8)$ and $60^{\circ}$, (b) $(0.8,0.6)$ and $60^{\circ}$.

$$
\begin{gathered}
f_{\mathrm{U} j}(t)=\frac{1}{2}\left[1-M_{1}^{\prime} \sin (\omega t)\right] \\
f_{\mathrm{Mj}}(t)=\frac{1}{2}\left[1+\frac{k_{1}}{k_{2}} M_{1}^{\prime} \sin (\omega t)-\frac{k_{3}}{k_{2}} M_{2}^{\prime} \sin (\omega t+\theta)\right] \\
f_{\mathrm{L} j}(t)=\frac{1}{2}\left[1+M_{2}^{\prime} \sin (\omega t+\theta)\right] .
\end{gathered}
$$

When the arm current flows through the arm, it will be coupled to the DC-side of SMs by switching actions. Then the average capacitor currents in upper, middle, and lower arms can be determined as follows:

$$
\begin{aligned}
i_{\mathrm{c} \_\mathrm{U} j}(t) & =f_{\mathrm{Uj} j}(t) i_{\mathrm{U} j}(t) \\
i_{\mathrm{c} \_\mathrm{M} j}(t) & =f_{\mathrm{M} j}(t) i_{\mathrm{M} j}(t) \\
i_{\mathrm{c} \_\mathrm{L} j}(t) & =f_{\mathrm{L} j}(t) i_{\mathrm{L} j}(t) .
\end{aligned}
$$

The capacitor voltage fluctuations can be easily calculated by integrating corresponding $\mathrm{AC}$ components in capacitor current, and the capacitor voltages in upper, middle, and lower arm can then be calculated as follows:

$$
\begin{aligned}
& u_{\mathrm{c} \__{-} \mathrm{U} j}(t)=U_{\mathrm{c} 0}+\frac{1}{C} \int i_{\mathrm{c} \__{-} \mathrm{Uj}}(t) \mathrm{d} t \\
& u_{\mathrm{c} \_\mathrm{M} j_{j}}(t)=U_{\mathrm{c} 0}+\frac{1}{C} \int i_{\mathrm{c} \_\mathrm{M} j_{j}}(t) \mathrm{d} t \\
& u_{\mathrm{c} \_ \text {Lj }}(t)=U_{\mathrm{c} 0}+\frac{1}{C} \int i_{\mathrm{c} \_ \text {Lj }}(t) \mathrm{d} t .
\end{aligned}
$$

The detailed expression of SM capacitor voltage ripples in the upper, middle, and lower arm can be derived in (B1)-(B6). As shown in Appendix B, the SM capacitor voltage ripples in 9A-MMC mainly consist of the first- and second-order ripple components as well. 


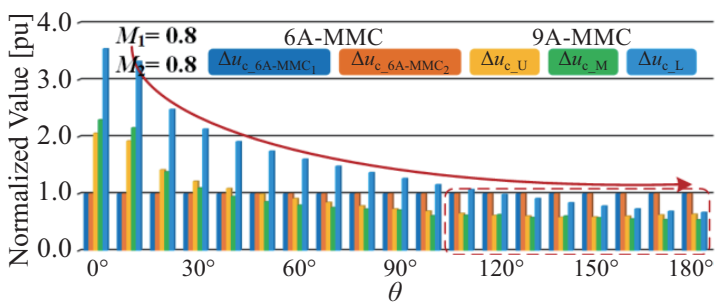

(a)

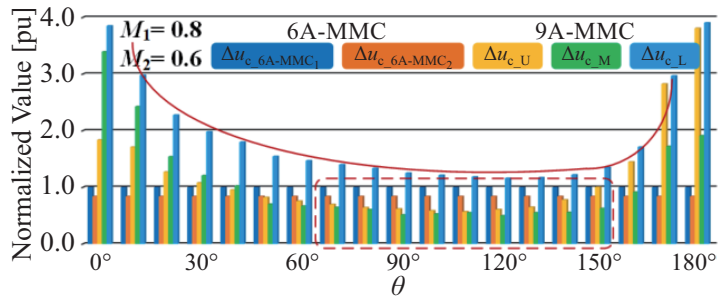

(b)

Fig. 8. Comparisons of the maximum capacitor voltage ripple between two 6A-MMCs and 9A-MMC when the modulation ratios are set as (a) 0.8 and 0.8 , (b) 0.8 and 0.6 .

Fig. 8 shows the comparisons of the maximum SM capacitor voltage ripple between 6A-MMC and 9A-MMC under different modulation ratios and relative phase angle, where only the first- and second-order capacitor voltage ripples are taken into account, and the maximum capacitor voltage ripple in 6A-MMC is used as a reference for comparison. In specific, Fig. 8(a) shows the comparison when the modulation ratios are set as 0.8 and 0.8 , and the relative phase angle changes from $0^{\circ}$ to $180^{\circ}$, Fig. 8 (b) shows the comparison with the relative phase angle varying from $0^{\circ}$ to $180^{\circ}$ when the modulation ratios are sets as 0.8 and 0.6 . As seen, the comparison of the maximum SM capacitor voltage ripple between 6A-MMC and 9A-MMC is similar to the comparison of the current stress of the semiconductor device in 6A-MMC and 9A-MMC. The maximum SM capacitor voltage ripple in 9A-MMC is not always larger than that in $6 \mathrm{~A}-\mathrm{MMC}$, which is also related to the operation scenario of 9A-MMC.

\section{Power Losses}

In [28], a calculation method of the power losses in 6A-MMC was presented, where the static-state losses including the conduction losses of switches and diodes are considered, and their off-state blocking losses are neglected. Besides, the switching losses including both the turn-on loss and the turnoff loss of switches and the recovery loss of fast recovery diodes are taken into accounts as well. The arm currents and switching signals can be firstly determined based on the specified operation condition and modulation method. Then, the conducting currents and voltage drop of all the switches and diodes will be obtained. The transient power loss can be achieved by multiplying the conducting current and voltage drop, then the conduction loss energy during one operation period can be obtained based on the integral of transient power
TABLE III

Simulation Parameters For 6A-MMC and 9A-MMC

\begin{tabular}{lcc}
\hline \hline Parameters & $6 \mathrm{~A}-\mathrm{MMC}$ & 9A-MMC \\
\hline SM capacitor voltage $U_{\mathrm{c}}$ & $600 \mathrm{~V}$ & $600 \mathrm{~V}$ \\
SM number per arm & $N=8$ & $k_{1}=7, k_{2}=8, k_{3}=5$ \\
DC-side voltage & $4.8 \mathrm{kV}$ & $6 \mathrm{kV}$ \\
SM capacitor $C$ & $1 \mathrm{mF}$ & $1 \mathrm{mF}$ \\
Fundamental frequency $f$ & $50 \mathrm{~Hz}$ & $50 \mathrm{~Hz}$ \\
Carrier frequency $f_{\mathrm{c}}$ & $5 \mathrm{kHz}$ & $5 \mathrm{kHz}$ \\
Arm inductor $L_{\text {arm }}$ & $2 \mathrm{mH}$ & $2 \mathrm{mH}$ \\
IGBT module & FF100R12RT4 & FF100R12RT4 \\
\hline \hline
\end{tabular}

loss. The conduction loss of the $i$ th SM can be expressed as follow:

$$
P_{\text {cond } \_i}(t)=\frac{1}{T} \int_{0}^{T}\left(u_{\mathrm{S} i 1} i_{\mathrm{S} i 1}+u_{\mathrm{S} i 2} i_{\mathrm{S} i 2}+u_{\mathrm{D} i 1} i_{\mathrm{D} i 1}+u_{\mathrm{D} i 2} i_{\mathrm{D} i 2}\right) \mathrm{d} t(48)
$$

where $u_{\mathrm{S} i 1}, u_{\mathrm{S} i 2}, u_{\mathrm{D} i 1}$, and $u_{\mathrm{D} i 2}$ are the voltage drops of $S_{i 1}, S_{i 2}, D_{i 1}$, and $D_{i 2}$, respectively, and $i_{\mathrm{S} i 1}, i_{\mathrm{S} i 2}, i_{\mathrm{D} i 1}$, and $i_{\mathrm{D} i 2}$ are the currents of $S_{\mathrm{x} i 1}, S_{\mathrm{x} i 2}, D_{\mathrm{xi} 1}$, and $D_{\mathrm{x} i 2}$, respectively.

The switching losses will be generated at each switching time, according to the switching states and current polarity at switching time, the switching behaviors and related energy losses can be derived. Then, accumulating the energy losses at every switching time one by one, the switching losses of all the switches and diodes will be finally obtained. The turn-on energies and the turn-off energies of switches and the recovery energies of fast recovery diodes at every switching time can be respectively given as follows:

$$
\begin{aligned}
& E_{\mathrm{S}_{-} \text {on }}=\frac{U_{\mathrm{c}} I_{\mathrm{S}_{\mathrm{on}}}}{U_{\mathrm{S}_{-} \mathrm{N}} I_{\mathrm{S}_{-} \mathrm{N}}} E_{\mathrm{S}_{\mathrm{S} \text { onN }}} \\
& E_{\mathrm{S}_{-} \text {off }}=\frac{U_{\mathrm{c}} I_{\mathrm{S}_{-} \text {off }}}{U_{\mathrm{S}_{-} \mathrm{N}} I_{\mathrm{S}_{-} \mathrm{N}}} E_{\mathrm{S} \_ \text {off }} \\
& E_{\text {D_rec }}=\frac{U_{\mathrm{c}} I_{\mathrm{D} \_ \text {rec }}}{U_{\mathrm{D} \_\mathrm{N}} I_{\mathrm{D} \_\mathrm{N}}} E_{\mathrm{D} \_ \text {rec N }}
\end{aligned}
$$

where $U_{\mathrm{S} \mathrm{N}}, I_{\mathrm{S} \mathrm{N}}$ and $U_{\mathrm{D} \mathrm{N}}, I_{\mathrm{D} \mathrm{N}}$ are the certain voltage and current of switches and diodes, $E_{\mathrm{S} \_ \text {onN }}$ and $E_{\mathrm{S} \_ \text {offN }}$ are the turnon and turn-off energies of switches at the certain voltage and current, $E_{\mathrm{D} \text { recN }}$ is the reverse recovery energy of diodes at the certain voltage and current, these data can be obtained from the datasheet, $I_{\mathrm{S} \text { on }}, I_{\mathrm{S} \text { off }}$, and $I_{\mathrm{D} \text { rec }}$ are the currents at switching time in practice.

The IGBT module adopted in both 6A-MMC and 9A-MMC is FF100R12RT4 produced by Infineon, and the design parameters of the converter are listed in Table III. The comparisons of power losses under different operation conditions between two 6A-MMCs and 9A-MMC are shown in Fig. 9. In specific, Fig. 9(a) shows the power losses comparisons with the relative phase angle changing from $0^{\circ}$ to $360^{\circ}$ when the modulation ratios are set as 0.8 and 0.8 . The power losses with the variation of relative phase angle when the modulation ratios are set 


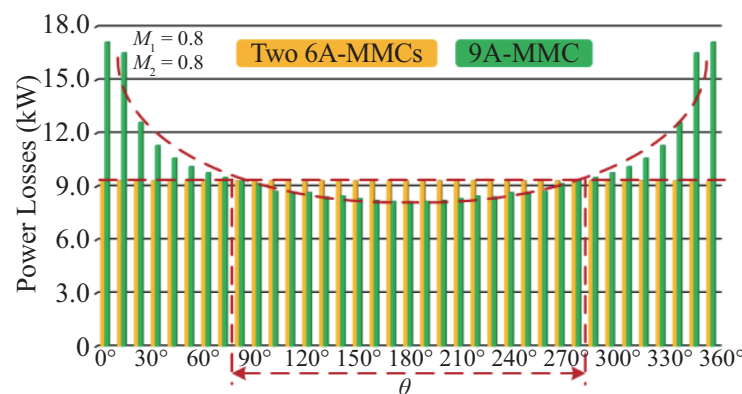

(a)

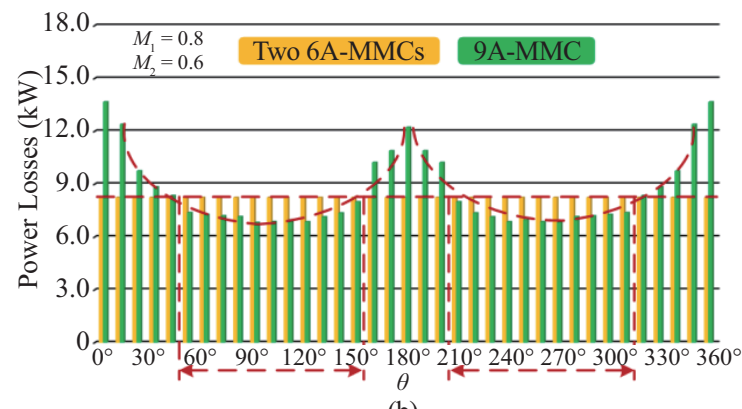

(b)

Fig. 9. Power losses of two 6A-MMCs and 9A-MMC with the variation of the relative phase angle $\theta$ when the modulation ratios are set as (a) 0.8 and 0.8 , (b) 0.8 and 0.6 .

as 0.8 and 0.6 are presented in Fig. 9(b). It is clear that the relative phase angle between two sets of output terminals does not affect the power losses of 6A-MMC, because two sets of output terminals in two 6A-MMCs can be decoupled by the common DC-link. However, the power losses of 9A-MMC are very relevant to the modulation ratios and the relative phase angle. Besides, the power losses of 9A-MMC are not always larger than that of two 6A-MMCs, 9A-MMC can obtain higher efficiency under some operation conditions. To investigate the relationship between the power factor angle and the power losses, Fig. 10 shows the power losses of two 6A-MMCs and 9A-MMC with different power factor angle, where the relative phase angle between two sets of output terminals is set as $60^{\circ}$. The power losses shown in Fig. 10(a) are under the modulation ratios are sets as 0.8 and 0.8 , while Fig. 10(b) shows the power losses when the modulation ratios are set as 0.8 and 0.6 . It is shown that the power losses of 6A-MMC and 9A-MMC can be decreased with the reduction of the output active power because the DC component of the arm currents can be decreased when the converter outputs less active power.

\section{Simulation Results}

To verify the mathematical analysis of the comparisons between 6A-MMC and 9A-MMC, the simulations of 6A-MMC and 9A-MMC is carried out in MATLAB/Simulink to power two identical inductive loads with $L=5 \mathrm{mH}$ and $R=30 \Omega$. The key simulation parameters are summarized in Table III, and the detailed control scheme can be found in [21].

The simulation results of the specific operation state that the

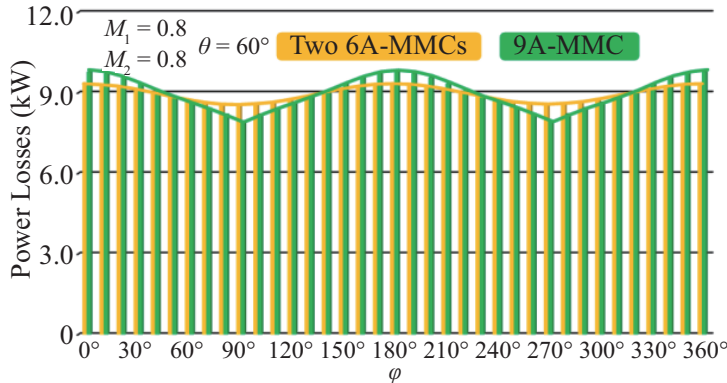

(a)

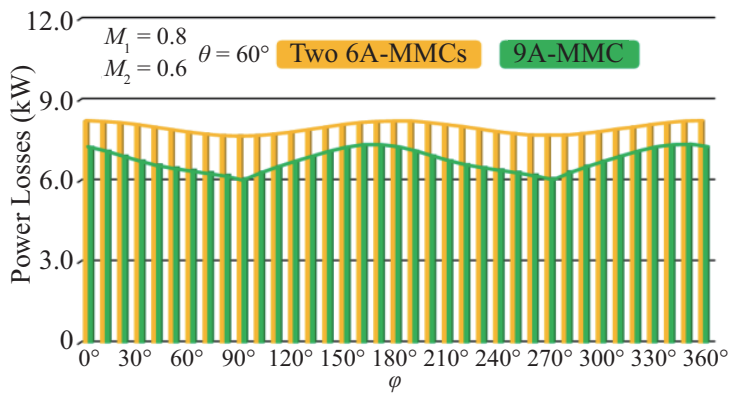

(b)

Fig. 10. Power losses of two 6A-MMCs and 9A-MMC with the change of the power factor angle $\varphi$ when the modulation ratios are set as (a) 0.8 and 0.8 , (b) 0.8 and 0.6 .

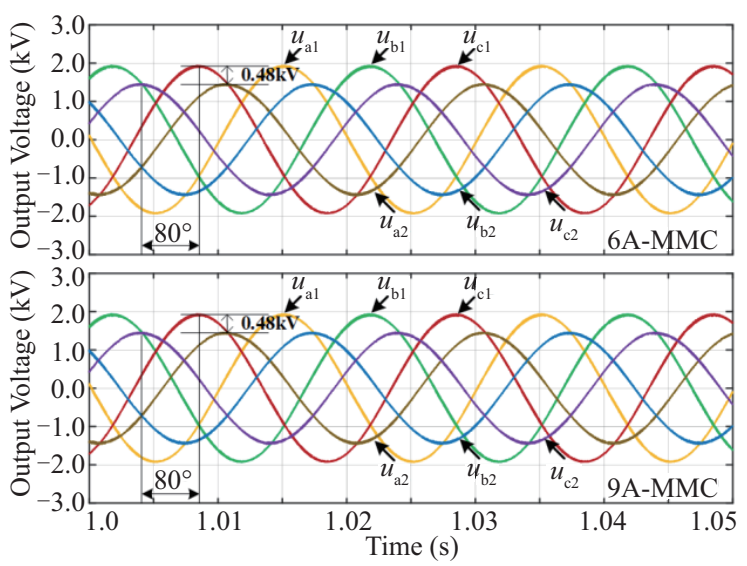

Fig. 11. Output voltages of two sets of output terminals in 6A-MMC and 9A-MMC.

modulation ratios are set as 0.8 and 0.6 , and the relative phase angle is set as $80^{\circ}$ are sequentially presented.

Two sets of output voltages of $6 \mathrm{~A}-\mathrm{MMC}_{1}, 6 \mathrm{~A}-\mathrm{MMC}_{2}$ and 9A-MMC $\left(u_{\mathrm{a} 1}, u_{\mathrm{b} 1}, u_{\mathrm{c} 1}\right.$ and $\left.u_{\mathrm{a} 2}, u_{\mathrm{b} 2}, u_{\mathrm{c} 2}\right)$ are shown in Fig. 11, and the two sets of output currents $\left(i_{\mathrm{a} 1}, i_{\mathrm{b} 1}, i_{\mathrm{c} 1}\right.$ and $\left.i_{\mathrm{a} 2}, i_{\mathrm{b} 2}, i_{\mathrm{c} 2}\right)$ are shown in Fig. 12. It is obvious that the 9A-MMC can output the standard sinusoidal waveforms like 6A-MMC. Fig. 13 presents the arm currents in 6A-MMC and 9A-MMC. As seen, the arm currents of the upper and lower arm in 6A-MMC $\left(i_{\mathrm{p}}\right.$, $i_{\mathrm{n}}$ ) are symmetrical, and the amplitude of them are identical. The arm currents of the upper, middle, and lower arm in 9A-MMC $\left(i_{\mathrm{U}}, i_{\mathrm{M}}\right.$, and $\left.i_{\mathrm{L}}\right)$ are asymmetrical. The SM capacitor 


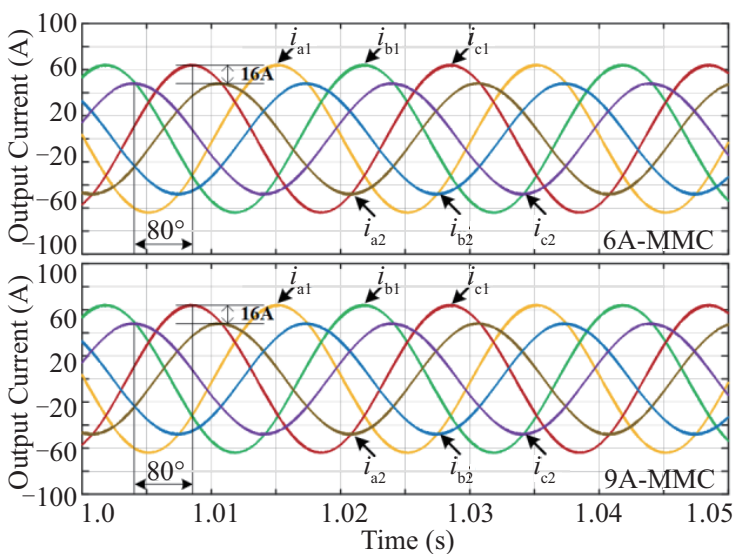

Fig. 12. Output currents of two sets of output terminals in 6A-MMC and 9A-MMC.

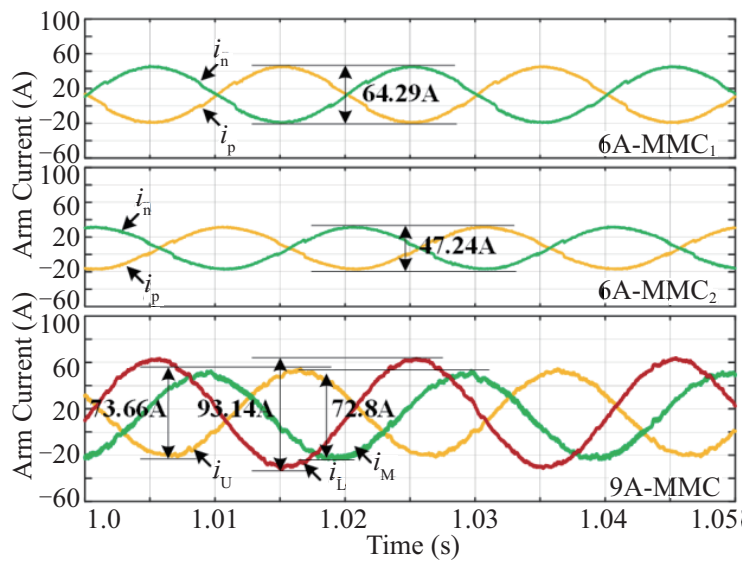

Fig. 13. Arm currents of 6A-MMC and 9A-MMC.

voltages of upper and lower arm in 6A-MMC $\left(u_{\mathrm{c} \_ \text {p }}, u_{\mathrm{c}_{\mathrm{n}} \mathrm{n}}\right)$ and the SM capacitor voltages of the upper, middle, and lower arm in 9A-MMC $\left(u_{\mathrm{c}_{\mathrm{U}}}, u_{\mathrm{c} \mathrm{M}}\right.$, and $\left.u_{\mathrm{c}_{\mathrm{L}}}\right)$ are displayed in Fig. 14. The maximum $\mathrm{SM}$ capacitor voltage ripple in $6 \mathrm{~A}-\mathrm{MMC}_{1}$ and $6 \mathrm{~A}-\mathrm{MMC}_{2}$ are $76.14 \mathrm{~V}$ and $66.15 \mathrm{~V}$, respectively, however, the maximum SM capacitor voltage ripple of upper, middle, and lower arm in 9A-MMC are $62.78 \mathrm{~V}, 58.79 \mathrm{~V}$, and 95.63 $\mathrm{V}$. The power losses of $6 \mathrm{~A}-\mathrm{MMC}_{1}, 6 \mathrm{~A}-\mathrm{MMC}_{2}$, and $9 \mathrm{~A}-\mathrm{MMC}$ are shown in Fig. 15. It is shown that the power losses of 9A-MMC are fewer than the total power losses of $6 \mathrm{~A}-\mathrm{MMC}_{1}$ and $6 \mathrm{~A}-\mathrm{MMC}_{2}$, and the power losses in the three phases of two 6A-MMCs and 9A-MMC are $8.24 \mathrm{~kW}$ and 7.15 $\mathrm{kW}$, respectively. Moreover, the comparisons between the theoretical analysis and simulation results including the current stress of semiconductor devices, the maximum SM capacitor voltage ripple, and the power losses of the 6A-MMC and 9A-MMC are summarized in Table IV.

\section{Conclusion}

This paper presented the comprehensive comparisons between 6A-MMC and 9A-MMC including the required SMs number, the current stress of the semiconductor device,

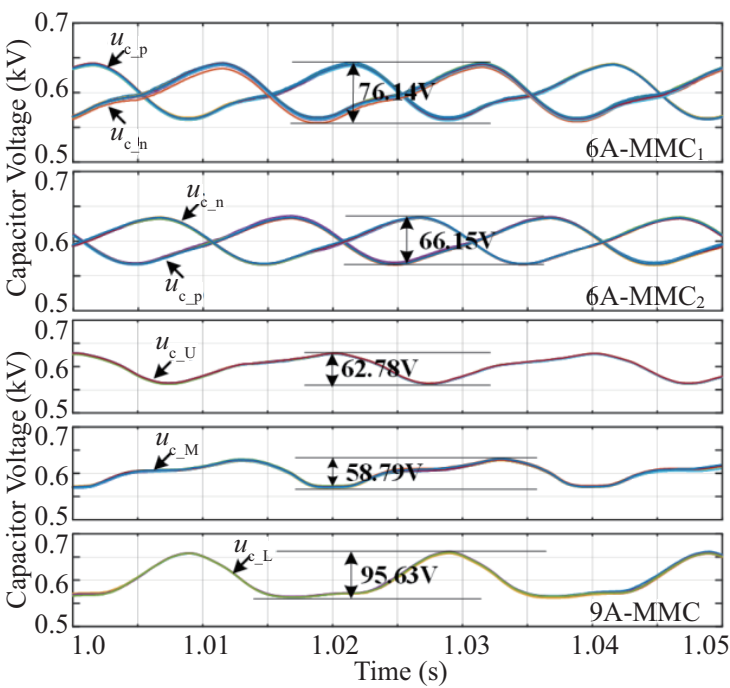

Fig. 14. SM capacitor voltages of 6A-MMC and 9A-MMC.

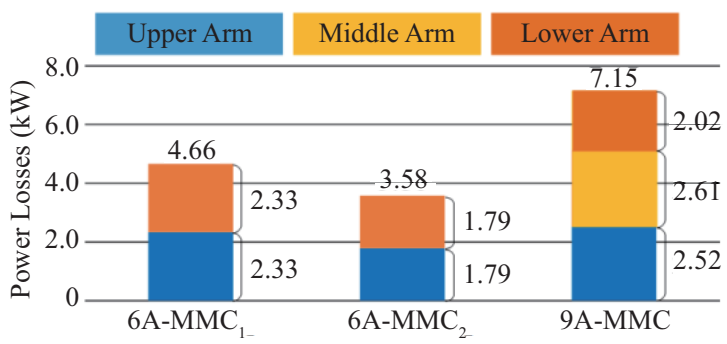

Fig. 15. Power losses of the 6A-MMC and 9A-MMC.

the maximum capacitor voltage ripple, and the whole system power losses. Compared with two 6A-MMCs, the number of required SMs in 9A-MMC can be reduced, especially when the modulation ratio difference between two sets of output terminals is large and the relative phase angle is small. Besides, the number of the corresponding SM capacitors, SM capacitor voltage sensors, and gate-drive circuits can also be reduced. The number of required SMs in 9A-MMC under the different frequency operation model is more than that under the common frequency operation model, which can weaken the advantage of 9A-MMC for reducing the components. The current stress of the semiconductor device and the maximum SM capacitor voltage ripple in 9A-MMC may be larger than that in 6A-MMC, but this is not universal. Under some specific operation conditions, the performance of the current stress of the semiconductor device and the maximum SM capacitor voltage ripple in 9A-MMC can be almost equivalent to the 6A-MMC. The power losses of 9A-MMC are also not always larger than that of two 6A-MMCs, and 9A-MMC even have higher efficiency under the specific operation conditions. The most obvious advantage of 9A-MMC is the reduction of the required components, but there are some performance tradeoffs like the other reduced-switch topology, and the performance tradeoffs of 9A-MMC are very relevant to the operation conditions. This paper identified these performance tradeoffs by the comparisons 
TABLE IV

Comparisons Between Theoretical Analysis and Simulation Results

\begin{tabular}{|c|c|c|c|c|c|c|c|c|}
\hline \multirow{2}{*}{$M_{1}=0.8, M_{2}=0.6, \theta=80^{\circ}$} & & \multicolumn{2}{|c|}{ Calculation } & \multicolumn{2}{|c|}{ Simulation } & & \multicolumn{2}{|c|}{ 9A-MMC } \\
\hline & & $6 \mathrm{~A}-\mathrm{MMC}_{1}$ & $6 \mathrm{~A}-\mathrm{MMC}_{2}$ & $6 \mathrm{~A}-\mathrm{MMC}_{1}$ & $6 \mathrm{~A}-\mathrm{MMC}_{2}$ & & Calculation & Simulation \\
\hline \multirow{2}{*}{$\begin{array}{l}\text { Current Stress of } \\
\text { Semiconductor Devices }\end{array}$} & Upper Arm & $63.01 \mathrm{~A}$ & $46.19 \mathrm{~A}$ & 64.29 & 47.24 & Upper Arm & $71.19 \mathrm{~A}$ & $73.66 \mathrm{~A}$ \\
\hline & Lower Arm & $63.01 \mathrm{~A}$ & $46.19 \mathrm{~A}$ & 64.29 & 47.24 & $\begin{array}{l}\text { Middle Arm } \\
\text { Lower Arm }\end{array}$ & $\begin{array}{r}70.3 \mathrm{~A} \\
90.92 \mathrm{~A}\end{array}$ & $\begin{array}{r}72.8 \mathrm{~A} \\
93.14 \mathrm{~A}\end{array}$ \\
\hline \multirow{2}{*}{$\begin{array}{l}\text { Maximum Capacitor } \\
\text { Voltage Ripple }\end{array}$} & Upper Arm & $74.96 \mathrm{~V}$ & $64.84 \mathrm{~V}$ & $76.14 \mathrm{~V}$ & $66.15 \mathrm{~V}$ & Upper Arm & $60.64 \mathrm{~V}$ & $62.78 \mathrm{~V}$ \\
\hline & Lower Arm & $74.96 \mathrm{~V}$ & $64.84 \mathrm{~V}$ & $76.14 \mathrm{~V}$ & $66.15 \mathrm{~V}$ & $\begin{array}{l}\text { Middle Arm } \\
\text { Lower Arm }\end{array}$ & $\begin{array}{l}57.21 \mathrm{~V} \\
92.39 \mathrm{~V}\end{array}$ & $\begin{array}{l}58.79 \mathrm{~V} \\
95.63 \mathrm{~V}\end{array}$ \\
\hline \multirow{3}{*}{ Power Losses } & Upper Arm & $2.33 \mathrm{~kW}$ & $1.79 \mathrm{~kW}$ & $2.38 \mathrm{~kW}$ & $1.83 \mathrm{~kW}$ & Upper Arm & $2.02 \mathrm{~kW}$ & $2.06 \mathrm{~kW}$ \\
\hline & Lower Arm & $2.33 \mathrm{~kW}$ & $1.79 \mathrm{~kW}$ & $2.38 \mathrm{~kW}$ & $1.83 \mathrm{~kW}$ & Middle Arm & $2.61 \mathrm{~kW}$ & $2.67 \mathrm{~kW}$ \\
\hline & Sum & $4.66 \mathrm{~kW}$ & $3.56 \mathrm{~kW}$ & $4.76 \mathrm{~kW}$ & $3.66 \mathrm{~kW}$ & $\begin{array}{c}\text { Lower Arm } \\
\text { Sum }\end{array}$ & $\begin{array}{l}2.52 \mathrm{~kW} \\
7.15 \mathrm{~kW}\end{array}$ & $\begin{array}{c}2.57 \mathrm{~kW} \\
7.3 \mathrm{~kW}\end{array}$ \\
\hline
\end{tabular}

between two 6A-MMC and 9A-MMC, which can be helpful to make the best use of the advantage of 9A-MMC.

\section{APPENDIx A}

The detailed expressions of the average absorbed power by three arms, and DC-side current are shown as follows:

$$
\begin{aligned}
P_{\mathrm{U} j}= & \frac{k_{1} U_{\mathrm{c}} I_{\mathrm{dc}}}{6}-\frac{\lambda I_{j 1} U_{j 1} \cos \varphi_{1}}{2}-\frac{I_{j 2} U_{j 1} \cos \left(\theta-\varphi_{2}\right)}{2} \\
& +\frac{\mu I_{j 2} U_{j 1} \cos \left(\theta-\varphi_{2}\right)}{2} \\
P_{\mathrm{M} j}= & \frac{k_{2} U_{\mathrm{c}} I_{\mathrm{dc}}}{6}-\frac{I_{j 1} U_{j 1} \cos \varphi_{1}}{2}+\frac{\lambda I_{j 1} U_{j 1} \cos \varphi_{1}}{2} \\
& +\frac{I_{j 2} U_{j 1} \cos \left(\theta-\varphi_{2}\right)}{2}-\frac{\mu I_{j 2} U_{j 1} \cos \left(\theta-\varphi_{2}\right)}{2} \\
& +\frac{I_{j 1} U_{j 2} \cos \left(\theta+\varphi_{1}\right)}{2}-\frac{\lambda I_{j 1} U_{j 2} \cos \left(\theta+\varphi_{1}\right)}{2} \\
& -\frac{I_{j 2} U_{j 2} \cos \varphi_{2}}{2}+\frac{\mu I_{j 2} U_{j 2} \cos \varphi_{2}}{2} \\
P_{\mathrm{L} j}= & \frac{k_{3} U_{\mathrm{c}} I_{\mathrm{dc}}}{6}-\frac{I_{j 1} U_{j 2} \cos \left(\theta+\varphi_{1}\right)}{2}+\frac{\lambda I_{j 1} U_{j 2} \cos \left(\theta+\varphi_{1}\right)}{2} \\
& -\frac{\mu I_{j 2} U_{j 2} \cos \varphi_{2}}{2} \\
& I_{\mathrm{dc}}=\frac{3\left(I_{j 1} U_{j 1} \cos \varphi_{1}+I_{j 2} U_{j 2} \cos \varphi_{2}\right)}{\left(k_{1}+k_{2}+k_{3}\right) U_{\mathrm{c}}}
\end{aligned}
$$

\section{APPENDIX B}

The detailed expressions of $u_{\mathrm{c} \_\mathrm{U} j \_1 \omega}, u_{\mathrm{c} \_\mathrm{U} j \_2 \omega}, u_{\mathrm{c} \_\mathrm{M} j \_1 \omega}, u_{\mathrm{c}_{\perp} \mathrm{M} \_2 \omega}$ $u_{\mathrm{c} \_\_\_\omega}$, and $u_{\mathrm{c} \_\perp_{j} \_\omega}$ are shown as follows:

$$
\begin{aligned}
u_{\mathrm{c} \_ \text {Lj } \_1 \omega}= & \frac{I_{\mathrm{dc}} M_{1} \cos (\omega t)}{6 \omega c}-\frac{\lambda I_{j 1} \cos \left(\omega t-\varphi_{1}\right)}{2 \omega c} \\
& -\frac{I_{j 2} \cos \left(\omega t+\theta-\varphi_{2}\right)}{2 \omega c}+\frac{\mu I_{\mathrm{a} 2} \cos \left(\omega t+\theta-\varphi_{2}\right)}{2 \omega c} \\
u_{\mathrm{c} \_ \text {L } \_2 \omega}= & \frac{\lambda I_{j 1} M_{1}^{\prime} \sin \left(2 \omega t-\varphi_{1}\right)}{8 \omega c}+\frac{(1-\mu) I_{\mathrm{a} 2} M_{1}^{\prime} \sin \left(2 \omega t+\theta-\varphi_{2}\right)}{8 \omega c}
\end{aligned}
$$

$$
\begin{aligned}
u_{c_{-} j_{j} \_1 \omega}= & -\frac{I_{\mathrm{dc}}\left[k_{1} M_{1}^{\prime} \cos (\omega t)-k_{3} M_{2}^{\prime} \cos (\omega t+\theta)\right]}{6 \omega c k_{2}} \\
& +\frac{(1-\lambda) I_{j 1} \cos \left(\omega t-\varphi_{1}\right)}{2 \omega c}-\frac{(1-\mu) I_{j 2} \cos \left(\omega t+\theta-\varphi_{2}\right)}{2 \omega c}
\end{aligned}
$$

$$
\begin{aligned}
& u_{\mathrm{c} \_\mathrm{M} j \_2 \omega}=\frac{(1-\lambda) I_{j 1} k_{1} M_{1}^{\prime} \sin \left(2 \omega t-\varphi_{1}\right)}{8 \omega c k_{2}} \\
& -\frac{(1-\mu) I_{j 2} k_{1} M_{1} \sin \left(2 \omega t+\theta-\varphi_{2}\right)}{8 \omega c k_{2}} \\
& -\frac{(1-\lambda) I_{j 1} k_{3} M_{2} \sin \left(2 \omega t+\theta-\varphi_{1}\right)}{8 \omega c k_{2}} \\
& +\frac{(1-\mu) I_{j 2} k_{3} M_{2} \sin \left(2 \omega t+2 \theta-\varphi_{2}\right)}{8 \omega c k_{2}} \\
& u_{c_{-} L_{-} \_1 \omega}=-\frac{I_{\mathrm{dc}} M_{2} \cos (\omega t+\theta)}{6 \omega c}+\frac{(1-\lambda) I_{j 1} \cos \left(\omega t-\varphi_{1}\right)}{2 \omega c} \\
& +\frac{\mu I_{j 2} \cos \left(\omega t+\theta-\varphi_{2}\right)}{2 \omega c} \\
& u_{\mathrm{c} \_ \text {L } \_2 \omega}=\frac{(1-\lambda) I_{j 1} M_{2} \sin \left(2 \omega t+\theta-\varphi_{1}\right)}{8 \omega c} \\
& +\frac{\mu I_{j 2} M_{2} \sin \left(2 \omega t+2 \theta-\varphi_{2}\right)}{8 \omega c}
\end{aligned}
$$

\section{REFERENCES}

[1] A. Lesnicar and R. Marquardt, "A new modular voltage source inverter topology," in Proceedings of European Power Electronics Conference, 2003, pp. $1-10$.

[2] A. Lesnicar and R. Marquardt, "An innovative modular multilevel converter topology suitable for a wide power range," in Proceedings of 2003 IEEE Bologna Power Tech Conference Proceedings, Bologna, Italy, 2003, pp. 1-6.

[3] M. Saeedifard and R. Iravani, "Dynamic performance of a modular multilevel back-to-back HVDC system," in IEEE Transactions on Power Delivery, vol. 25, no. 4, pp. 2903-2912, Oct. 2010.

[4] A. Nami, J. Liang, F. Dijkhuizen, and G. D. Demetriades, "Modular multilevel converters for HVDC applications: Review on converter cells and functionalities," in IEEE Transactions on Power Electronics, vol. 30, no. 1, pp. 18-36, Jan. 2015.

[5] M. Guan and Z. Xu, "Modeling and control of a modular multilevel 
converter-based HVDC system under unbalanced grid conditions," in IEEE Transactions on Power Electronics, vol. 27, no. 12, pp. 48584867, Dec. 2012.

[6] X. Liu, J. Lv, C. Gao, Z. Chen, and S. Chen, "A novel STATCOM based on diode-clamped modular multilevel converters," in IEEE Transactions on Power Electronics, vol. 32, no. 8, pp. 5964-5977, Aug. 2017.

[7] H. P. Mohammadi and M. T. H. Bina, "A transformerless mediumvoltage STATCOM topology based on extended modular multilevel converters," in IEEE Transactions on Power Electronics, vol. 26, no. 5, pp. 1534-1545, May 2011.

[8] Q. Hao, J. Man, F. Gao, and M. Guan, "Voltage limit control of modular multilevel converter based unified power flow controller under unbalanced grid conditions," in IEEE Transactions on Power Delivery, vol. 33, no. 3, pp. 1319-1327, Jun. 2018.

[9] B. Tai, C. Gao, X. Liu, and Z. Chen, "A novel flexible capacitor voltage control strategy for variable-speed drives with modular multilevel converters," in IEEE Transactions on Power Electronics, vol. 32, no. 1, pp. 128-141, Jan. 2017.

[10] B. Li, S. Zhou, D. Xu, S. J. Finney, and B. W. Williams, "A hybrid modular multilevel converter for medium-voltage variable-speed motor drives," in IEEE Transactions on Power Electronics, vol. 32, no. 6, pp. 4619-4630, Jun. 2017.

[11] M. A. Perez, S. Bernet, J. Rodriguez, S. Kouro, and R. Lizana, "Circuit topologies, modeling, control schemes and applications of modular multilevel converters," in IEEE Transactions on Power Electronics, vol. 30, no. 1, pp. 4-17, Mar. 2014

[12] A. Dekka, B. Wu, R. L. Fuentes, M. Perez, and N. R. Zargari, "Evolution of topologies, modeling, control schemes, and applications of modular multilevel converters," in IEEE Journal of Emerging and Selected Topics in Power Electronics, vol. 5, no. 4, pp. 1631-1656, Dec. 2017.

[13] S. Debnath, J. Qin, B. Bahrani, M. Saeedifard, and P. Barbosa, "Operation, control, and applications of the modular multilevel converter: A review," in IEEE Transactions on Power Electronics, vol. 30, no. 1, pp. 37-53, Jan. 2015.

[14] Y. S. Kumar and G. Poddar, "Control of medium-voltage AC motor drive for wide speed range using modular multilevel converter," in IEEE Transactions on Industrial Electronics, vol. 64, no. 4, pp. 2742-2749, Apr. 2017.

[15] A. A. Elserougi, A. S. Abdel-Khalik, A. M. Massoud, and S. Ahmed, "A nine-arm modular multilevel converter (9A-MMC) for six-phase medium voltage motor drives," in Proceedings of IECON 2015 41st Annual Conference of the IEEE Industrial Electronics Society, Yokohama, Japan, 2015, pp.1735-1740.

[16] A. A. Elserougi, A. S. Abdel-Khalik, A. M. Massoud, and S. Ahmed, "An asymmetrical six-phase induction motor drive based on ninearm Modular Multilevel Converter (9AMMC) with circulating current suppression," in Proceedings of 2015 4th International Conference on Electric Power and Energy Conversion Systems (EPECS), Sharjah, United Arab Emirates, 2015, pp. 1-6.

[17] M. S. Diab, G. P. Adam, B. W. Williams, A. M. Massoud, and S. Ahmed, "Quasi two-level PWM operation of a nine-arm modular multilevel converter for six-phase medium-voltage motor drives," in Proceedings of 2018 IEEE Applied Power Electronics Conference and Exposition (APEC), San Antonio, TX, USA, 2018, pp. 1641-1648.

[18] F. Qin, F. Gao, T. Xu, D. Niu, and Z. Ma, "A unified power flow controller with nine-arm modular multilevel converter," in Proceedings of 2018 IEEE Energy Conversion Congress and Exposition (ECCE), Portland, OR, USA, 2018, pp. 2581-2587.

[19] J. Fu, B. Zhang, and D. Qiu, "A novel nine-arm modular multilevel converter," in Proceedings of IECON 2014 - 40th Annual Conference of the IEEE Industrial Electronics Society, Dallas, TX, USA, 2014, pp. 4528-4533.

[20] F. Qin, F. Gao, T. Xu, D. Niu, and Z. Ma, "A control scheme of ninearm modular multilevel converter," in Proceedings of 2018 IEEE Energy Conversion Congress and Exposition (ECCE), Portland, OR, USA, 2018, pp. 2143-2148.

[21] F. Qin, F. Gao, C. Zhang, "Operational analyses and control scheme of nine-arm modular multilevel converter," in IEEE Transactions on Power
Electronics, vol. 35, no. 4, pp. 3416-3433, Apr. 2020.

[22] T. Kominami and Y. Fujimoto, "A novel nine-switch inverter for independent control of two three-phase loads," in Proceedings of 2007 IEEE Industry Applications Annual Meeting, New Orleans, LA, USA, 2007, pp. 2346-2350.

[23] F. Gao, L. Zhang, D. Li, P. C. Loh, Y. Tang, and H. Gao, "Optimal pulsewidth modulation of nine-switch converter," in IEEE Transactions on Power Electronics, vol. 25, no.9, pp. 2331-2343, Sep. 2010.

[24] Z. Qin, P. C. Loh, A. Bahman, and F. Blaabjerg, "Evaluation of current stresses in nine-switch energy conversion systems," in IET Power Electronics, vol. 7, no. 11, pp. 2877-2886, Nov. 2014.

[25] K. Ali, P. Das, and S. K. Panda, "A special application criterion of the nine-switch converter with reduced conduction loss," in IEEE Transactions on Industrial Electronics, vol. 65, no. 4, pp. 2853-2862, Apr. 2018

[26] Z. Qin, P. C. Loh, and F. Blaabjerg, "Application criteria for nine-switch power conversion systems with improved thermal performance," in IEEE Transactions on Power Electronics, vol. 30, no. 8, pp. 4608-4620, Aug. 2015.

[27] Q. Song, W. Liu, X. Li, H. Rao, S. Xu, and L. Li, "A steady-state analysis method for a modular multilevel converter," in IEEE Transactions on Power Electronics, vol. 28, no. 8, pp. 3702-3713, Aug. 2013.

[28] J. Li, X. Zhao, Q. Song, H. Rao, S. Xu, and M. Chen, "Loss calculation method and loss characteristic analysis of MMC-based VSC-HVDC system," in Proceedings of 2013 IEEE International Symposium on Industrial Electronics, Taipei, Taiwan, 2013, pp. 1-6.

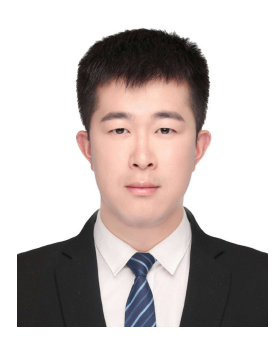

Futian Qin received B.Eng. degree in electrical engineering from Shandong Agricultural University, Tai' an, China, in 2015. Since 2015, he has been working toward $\mathrm{Ph} . \mathrm{D}$. degree in electrical engineering with Shandong University, Jinan, China. His research interests include modular multilevel converter (MMC), Nine-arm MMC, HVDC, and FACTS. From December 2019 to June 2020, he was a Research Assistant at Energy Research Institute @ Nanyang Technological University, Singapore.

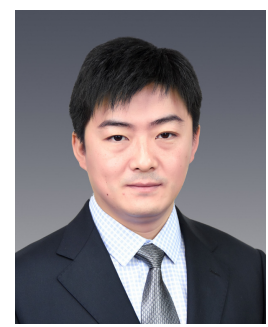

Feng Gao received the B.Eng. and M.Eng. degrees in electrical engineering from Shandong University, Jinan, China, in 2002 and 2005, respectively, and the Ph.D. degree from the School of Electrical and Electronic Engineering, Nanyang Technological University, Singapore, in 2009. From 2008 to 2009, he was a research fellow in Nanyang Technological University. Since 2010, he joined School of Electrical Engineering, Shandong University, where he is currently a professor. From September 2006 to February 2007, he was a visiting scholar at the Institute of Energy Technology, Aalborg University, Aalborg, Denmark.

Dr. Gao was the recipient of the IEEE Industry Applications Society Industrial Power Converter Committee Prize for a paper published in 2006 and 2017 IEEE Power Electronics Transactions Second Prize Paper Award, and he is now serving as the Associate Editors of IEEE Transactions on Power Electronics and CPSS Transcations on Power Electronics and Applications. 


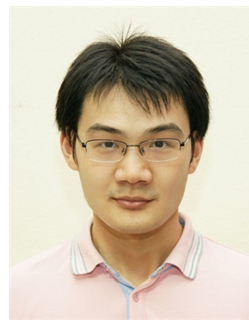

Yi Tang received the B.Eng. degree in electrical engineering from Wuhan University, Wuhan, China, in 2007 and the M.Sc. and Ph.D. degrees from the School of Electrical and Electronic Engineering, Nanyang Technological University, Singapore, in 2008 and 2011, respectively.

From 2011 to 2013, he was a Senior Application Engineer with Infineon Technologies Asia Pacific, Singapore. From 2013 to 2015, he was a Postdoctoral Research Fellow with Aalborg University, Aalborg, Denmark. Since March 2015, he has been with Nanyang Technological University, Singapore as an Assistant Professor. He is the Cluster Director of the Advanced Power Electronics Research Program at the Energy Research Institute, Nanyang Technological University.

Dr. Tang was a recipient of the Infineon Top Inventor Award in 2012, the Early Career Teaching Excellence Award in 2017, and four IEEE Prize Paper Awards. He serves as an Associate Editor for the IEEE Transactions on Power Electronics (TPEL) and the IEEE Journal of Emerging and Selected Topics in Power Electronics (JESTPE).

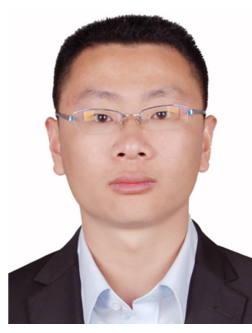

Jinyu Wang received his B.Eng. degree in electrical engineering and M.Eng. degree in power electronics from Jilin University, Changchun, China, in 2010 and 2013, respectively, and the Ph.D. degree in power system from Shandong University, Jinan, China, in 2017. From July 2017 to June 2019, he was a Postdoctoral Research Fellow with Rolls-Royce Cooperate Lab @ Nanyang Technological University, Singapore. Since June 2019, he has been with Energy Research Institute @ Nanyang Technological University, Singapore, as a Research Fellow.

His current research interests include power electronics, multilevel converters, renewable energy generation and integration techniques as well as stability analysis and control of modular multilevel converter based HVDC.

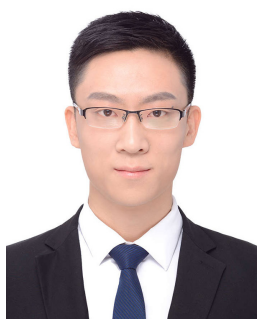

Tao Xu received his B.Eng. degree and Ph.D degree in electrical engineering from Shandong University, Jinan, China, in 2014 and 2019. From September 2017 to September 2018, he was a Visiting Scholar at the Institute of Energy Technology, Aalborg University, Aalborg, Denmark. Since 2019, he joined School of Electrical Engineering, Shandong University, where he is currently a postdoc. Tao Xu was the recipient of the IEEE Power Electronics Transactions Second Prize Paper Award in 2017 and the IEEE PELS Prize Ph.D. Thesis Talk in 2019. His research interests are parallel inverters, power quality and modulation methods. 